\title{
Infekt bei intramedullärem Implantat
}

Matthias Militz

\section{Einleitung}

Mit der klinischen Etablierung der Marknagelosteosynthese durch Kuentscher [1] eröffneten sich neue Möglichkeiten für die Frakturbehandlung an langen Röhrenknochen. Ohne wesentliche iatrogene Weichteilkompromittierung konnte ein frakturierter Röhrenknochen über den Marknagel im Markraum stabilisiert und zur Ausheilung gebracht werden. Mit den Anfängen der systematischen Frakturforschung und Entwicklung von Implantaten durch die Arbeitsgemeinschaft für Osteosynthesefragen $(A O)$ geriet der Marknagel als Osteosyntheseverfahren vorübergehend aus dem Fokus, um mit Entwicklung der verriegelten Marknägel neue Indikationsspektren zu erobern, da mit der Plattenosteosynthese zwar eine anatomische Reposition möglich war, durch die Beeinflussung der Durchblutung jedoch die Infektionsraten stark anstiegen und die sog. „biologische“ Osteosynthese zur Schonung der Weichteilsituation angestrebt wurde. Mit dem Marknagel war die Stabilisierung auch in gedeckter Technik möglich und eröffnete diesem Osteosyntheseverfahren neue Impulse.

Heute sind Marknägel in verschiedenen Varianten an allen langen Röhrenknochen als Osteosyntheseverfahren etabliert und verfügbar. Das weite Indikationsspektrum und die demografischen und gesundheitspolitischen Entwicklungen führen dazu, dass die Indikationen für operative Frakturbehandlungen weiter gestellt sind und damit auch die absolute Zahl der Infektionen ansteigt, obwohl das Infektionsrisiko insgesamt eher als niedrig einzustufen ist.

Damit ergibt sich für die Behandlung von Infektionen bei intramedullärem Implantat die Herausforderung, unter Erhalt der biologischen Voraussetzungen für die Frakturheilung den Infekt zu sanieren und eine funktionell stabile Konsolidierung des Knochens zu erreichen.

Die Besonderheiten bei der Diagnostik und Behandlung von Infektionen bei intramedullärem Implantat sollen in diesem Beitrag dargestellt und eigene Erfahrungen beschrieben werden.

\section{Hauptteil}

\section{Inzidenz}

Zuverlässige Angaben über die Häufigkeit von Infektionen bei intramedullärem Implantat existieren nicht. Es ist bekannt, dass die Häufigkeit von postoperativen Infektionen nach Osteosynthese von Frakturen der langen Röhrenknochen zwischen 0 und 7\% [2-12] variiert. Die Unterschiede ergeben sich aus zahlreichen Faktoren, welche die Entstehung einer postoperativen Infektion begünstigen. So ist das Risiko bei Kompromittierung des Immunsystems, z. B. beim Polytrauma mit großem Blutverlust ebenso wie durch Erkrankungen, welche die Durchblutung beeinträchtigen, wie Nikotinkonsum, PAVK und Diabetes mellitus, erhöht [13-15]. Dabei ist das Infektionsrisiko auch bei geschlossenen Frakturen erhöht. Wird eine geschlossene Fraktur im Rahmen der Operation freigelegt, so wird diese in eine offene umgewandelt und hat ebenfalls ein erhöhtes Infektionsrisiko $[16,17]$.

Die anatomische Region hat aufgrund unterschiedlicher Perfusionsverhältnisse ebenfalls Einfluss auf das Infektionsrisiko. Infektionen an der oberen Extremität sind wesentlich seltener und beeinträchtigen den Patienten in seiner Lebensqualität in aller Regel weniger.

Die Marknagelosteosynthese ist das ideale Implantat für die Stabilisierung von Frakturen der langen Röhrenknochen. Neben der optimalen biomechanischen Situation wird das Weichteiltrauma durch die Operation gering gehalten. Diese Vorteile sind im Falle einer Infektion jedoch auch die Faktoren, welche die Diagnostik und Therapie zu einer besonderen Herausforderung gestalten.

\section{Diagnostik}

Die Diagnostik der Infektion bei intramedullärem Implantat entspricht den Prinzipien der septischen Chirurgie und wird wesentlich vom klinischen Verlauf, den Laborbefunden und der Bildgebung bestimmt. Beweisend für die Infektion sind der positive mikrobiologische und der histologische Befund. Allerdings stehen diese Informationen erst postoperativ zur Verfügung.

Anamnestisch sind offene Frakturen, lange OP-Zeiten, Wundheilungsstörungen, „Allergien“, Antibiotikagabe etc. hinweisend auf eine Infektion. 
Klinisch reicht das Erscheinungsbild der Infektion bei intramedullärem Implantat von Schmerzen und geringen lokalen Auffälligkeiten bis hin zum septischen Schock.

Während die akute posttraumatische/-operative Infektion eher durch einen markanten Verlauf mit Fieber, Schmerzen und akuten Infektzeichen gekennzeichnet ist, verläuft die chronische Infektion überwiegend schleichend, zeitweise undulierend und mit weniger stark ausgeprägten Infektzeichen. Bei Verdacht auf hämatogene Infektion ist die Fokussuche/-ausschluss erforderlich.

Laborchemisch ist im klinischen Alltag für die Diagnostik von muskuloskeletalen Infektionen der CRP-Wert richtungweisend, wenn auch nicht beweisend $[18,19]$.

In der akuten Situation eines Infektverdachts bestimmen der klinische Verlauf und der Lokalbefund die OP-Indikation. Beim Verdacht auf eine akute Infektion ist damit bereits die Indikation zur Revision gestellt. Hier helfen bildgebende Verfahren wenig weiter. Bei begründetem Verdacht kann mittels CT oder besser MRT eine mögliche Abszessformation in den Weichteilen detektiert werden und für die präoperative Planung hilfreich sein, wird aber mitunter in der Aussagefähigkeit durch Artefakte von seiten des Implantates limitiert.

Bei chronischen Infektionen besteht die Herausforderung in der Detektion des Sequesters/Infektfokus bei liegendem Implantat. Parallel sind für die Planung der Sanierung und Rekonstruktion die Abklärung und Optimierung der Durchblutungssituation, der Stabilität bzw. das Ausmaß des knöchernen Defektes essenziell.

Bei klinischem Verdacht auf eine Infektion bei intramedullärem Implantat gelingt die Sicherung der Diagnose oft erst postoperativ, sodass ein mehrzeitiges Konzept zur Sanierung und Rekonstruktion naheliegend ist.

\section{Therapie}

Die Grundsätze der septischen Chirurgie mit Sequestrektomie, Implantatentfernung und Stabilisierung gelten auch bei Infektionen mit intramedullärem Implantat.

Während die Sequestrektomie und die Implantatentfernung an allen Röhrenknochen ähnlich umzusetzen sind, erfordert die Stabilisierung eines instabilen infizierten Femurs besonderer Aufmerksamkeit. Vorübergehend kann eine externe Stabilisierung eines Unterschenkels oder Armes auch im Cast o. ä. erfolgen, ohne dass eine dramatische Beeinträchtigung des Patienten resultiert. Ganz anders am Femur. Weder durch Cast noch durch Fixateur externe ist eine komfortable und zufriedenstellende Stabilisierung möglich. Neben der Weichteilkompromittierung und der limitierten Stabilität kommt hinzu, dass der Markraum durch Fixateurpins verlegt ist und für das Débridement/Sequestrektomie nicht zugänglich ist. Zur Be- wältigung dieser Situation bietet sich der Marknagel aus mehreren Gründen als Wechselimplantat für die Sanierung bei Femurinfektionen an.

Nach Entfernung des Marknagels ist der gesamte Knochen für die Sequestrektomie zugänglich, ohne eine weitere Traumatisierung des umgebenden Gewebes zu verursachen. Nach der Entnahme von mikrobiologischen und histologischen Proben zur Diagnosesicherung erfolgt die Sequestrektomie und Markraumüberbohrung, bis der Bohrer Widerstand beim Bohren erreicht, in aller Regel 0,5 bis $1 \mathrm{~mm}$ größer als das entfernte Implantat. Anschließend Jetlavage des Markraumes und Desinfektion des Situs.

Zur Reduktion der Keimlast erfolgt nun der Wechsel der Abdeckung, Instrumente und Handschuhe. Nach lokaler Applikation von Antibiotika im Markraum erfolgt die erneute Stabilisierung mit einem Implantat der gleichen Dimension des vorher einliegenden Implantates, um eine zusätzliche Destruktion der Knochensubstanz zu limitieren. Abhängig vom Weichteilbefund erfolgt der Wundverschluss mit oder ohne Vakuumversiegelung. Es resultiert eine lagerungsstabile Extremität. Begleitend erfolgt die kalkulierte Antibiotikatherapie. Diese Prozedur wird bis zum Erhalt von negativen mikrobiologischen Proben in ca. wöchentlichen Intervallen bis zu 3 Mal vorgenommen. Nach der 1. Revision erfolgt eine CT-Kontrolle, ggf. als Angio-CT, um noch verbliebene Sequester zu detektieren. Besonders bei ehemaligen Trümmerfrakturen ist durch das runde Bohrloch ein exzentrisch gelegener Sequester nicht zu erreichen und bedarf der gezielten Entfernung. Neben der Beurteilung der Durchblutungssituation bei den oft unphysiologischen Verhältnissen kann die Planung eventueller plastisch-chirurgischer Operationen mittels CT-Angiografie vorbereitet werden.

Infektionen bei intramedullärem Implantat an Tibia, Humerus, Ulna oder Radius erfordern ebenfalls die Implantatentfernung, Sequestrektomie und Aufbohrung des Markraumes. Obligat ist auch hier die differenzierte Entnahme von Gewebeproben für die mikrobiologische und histologische Untersuchung zur Sicherung der Diagnose. Bei der Jetlavage sollte auf einen ausreichenden Abfluss der Spülflüssigkeit und Schonung der Weichteile geachtet werden. Nach dem Wechsel der Abdeckung und Instrumente hat sich zur Infektberuhigung die Applikation von topisch wirkenden Antibiotika bewährt. Während sich bei Verwendung von antibiotikahaltigen Ketten sowohl die Applikation als auch die Entfernung schwierig gestalten kann, erscheint die Verwendung von antibiotikahaltigen Platzhaltern in Form von Knochenzementnägeln einfacher in der Handhabung. Abhängig vom Weichteildefekt erfolgt der Wundverschluss oder die Vakuumversiegelung. Nach Vorliegen des mikrobiellen Befundes und der Histologie kann die Antibiotikatherapie adaptiert werden. Temporär kann am Unterschenkel eine Immobi- 
lisierung im Cast, an der oberen Extremität im GilchristVerband vorgenommen werden. Postoperativ erfolgt befundabhängig die CT-Untersuchung zur Beurteilung der Sequestrektomie, bei erforderlicher plastisch-chirurgischer Behandlung als CT-Angio. Im Intervall wird die Optimierung der Perfusionsverhältnisse vorgenommen und die Knochen- und Weichteilrekonstruktion geplant.

\section{Rekonstruktion}

Nach Eradikation des Infektherdes hängt das Verfahren der Rekonstruktion vom Ausmaß der Defektstrecke, der Lokalisation, dem begleitenden Weichteildefekt, Nebenerkrankungen, dem Patientenwunsch und vielen weiteren Faktoren ab.

Bei kurzen Defekten bietet sich die Verkürzungsresektion und bei keimfreiem Situs die erneute Marknagelosteosynthese an. Am Unterschenkel ist dieses Verfahren nur möglich, wenn auch die Fibula verkürzt wird oder nicht sperrt. Bei Erhalt der Unterschenkellänge können Defekte bis ca. $3 \mathrm{~cm}$ auch bei liegendem Marknagel mit einer Spongiosaplastik aufgefüllt oder mit einem Segmenttransport bei liegendem Marknagel rekonstruiert werden. Defekte mit einem Ausmaß von mehr als ca. $5 \mathrm{~cm}$ können relativ zuverlässig mit dem Segmenttransport über Marknagel oder Fixateur externe behandelt werden. [20-22] Nach anfänglichen Erfolgen der Defektrekonstruktion mit der Masquelet-Technik scheint dieses Verfahren für größere Defekte (> $5 \mathrm{~cm}$ ) nur bedingt geeignet $[23,24]$.

Während sich der Ringfixateur durch die Variabilität, Stabilität und beim Hexapod-Fixateur auch durch eine 3-dimensionale Korrekturmöglichkeit auszeichnet, erscheint der Monorail-Fixateur komfortabler, aber bei anspruchsvoller OP-Technik nicht bei allen Situationen vorteilhaft. Bei der Rekonstruktion von Diaphysendefekten mit einer vaskularisierten Fibula ist eine sichere Stabilisierung in aller Regel mit Fixateur externe oder Platte erforderlich. Eine belastbare Stabilität ist nach einem Zeitraum von ca. 1-2 Jahren zu erwarten. Bei der Stabilisierung mit einer Platte ist immer zu berücksichtigen, dass neben der kompromittierten Markraumdurchblutung dann zusätzlich auch die periostale Durchblutung gestört ist und ein zusätzlicher Zugang mit entsprechender Weichteilschädigung erforderlich ist. Bei geplantem extramedullärem Zugang aus anderen Gründen (z. B. Segmentresektion) spielt dieser Aspekt eine untergeordnete Rolle.

Semizirkuläre Knochendefekte bis zu 2/3 der Zirkumferenz können bei ausreichender Stabilität und Durchblutungssituation mit Spongiosa, Knochenersatzmaterial oder vaskularisiertem Knochen überbrückt werden. Die Entscheidung zur Auswahl eines erfolgversprechenden Verfahrens hängt von zahlreichen Faktoren ab, ist immer individuell und sollte den Prinzipien der septischen Chirurgie entsprechen.
Die Infektion bei intramedullärem Implantat an der unteren Extremität bedeutet immer eine erhebliche Beeinträchtigung der Mobilität des Patienten. Abhängig von den Nebenerkrankungen und der Compliance resultiert oft die Rollstuhlabhängigkeit für den Zeitraum der Sanierung.

Die Therapie von Infektionen bei intramedullärem Implantat an Humerus, Ulna und Radius gestaltet sich demgegenüber vergleichsweise einfach, sofern ausreichend knöcherne Substanz für die Rekonstruktion vorhanden ist.

Abhängig vom Ausmaß der Infektion, der resultierenden Defektsituation, der Durchblutungsverhältnisse, der Nebenerkrankungen und dem Patientenwunsch kann auch die Amputation als Therapieoption ein geeignetes Verfahren zur Infektbehandlung darstellen.

\section{Schlussfolgerungen}

Infektionen bei intramedullärem Implantat stellen trotz moderner Implantate, hochwirksamer Antibiotika und gewebeschonender OP-Verfahren immer noch eine interdisziplinäre Herausforderung dar. Der Schlüssel zur erfolgreichen Behandlung liegt in der radikalen chirurgischen Sanierung des Infektionsherdes. Im Vorfeld operativer Maßnahmen ist die Optimierung der Durchblutung und der Nebenerkrankungen anzustreben. Da der Markraum offen kaum zugänglich ist, erfolgt die Sequestrektomie über Aufbohren der Markhöhle und ggf. offene Sequestrektomie. Tibia und obere Extremität lassen sich für ein implantatfreies Intervall gut mit einem Cast/GilchristVerband ruhigstellen, am Femur erscheint der Marknagelwechsel für die Stabilisierung im Intervall ein geeigneter Kompromiss zwischen immobilisierender Instabilität und Herdsanierung zu sein. Nach Sicherung der Diagnose mit mikrobiologischen und histologischen Proben erfolgt die Rekonstruktion des Knochens und ggf. der Weichteile. Abhängig vom Ausmaß des Defektes, der Lokalisation, der Begleitumstände und des Patientenwunsches kommen die Spongiosaplastik, Masquelet-Technik, die Verkürzungsosteotomie, die Segmentresektion mit anschließendem Segmenttransport, der freie Fibulatransfer bis hin auch zur Amputation jeweils als das individuell geeignete Verfahren zur Anwendung.

Zur Therapie dieses multifaktoriellen Krankheitsbildes ist ein interdisziplinär angestimmtes Therapiekonzept unabdingbar.

Interessenkonflikt

Teilhaber sBone-GmbH, Beratung Fa. BBraun, Beratung Berufsgenossenschaften, Beratung Fa. BoneAlive 
Über die Autoren

\section{Matthias Militz}

Dr., BG Unfallklinik Murnau

\section{Korrespondenzadresse}

\section{Dr. Matthias Militz}

BG Unfallklinik Murnau

Prof.-Küntscher-Straße 8

82418 Murnau

Matthias.Militz@bgu-murnau.de

\section{Literatur}

[1] Kuentscher G. Intramedullary splinting. Med Bull U S Army Force Europe Theater Off Theater Chief Surg 1947; 3: 5-8

[2] Vecsei V, Heinz T. [The interlocking nail for long comminuted and compound fractures of the femur and tibia. Technique and results]. Unfallchirurg 1990; 93: 512-518

[3] Sehat K, Baker RP, Pattison G et al. The use of the long gamma nail in proximal femoral fractures. Injury 2005; 36: 1350-1354

[4] Schratz W, Worsdorfer O, Klockner C et al. [Treatment of humeral shaft fracture with intramedullary procedures (Seidel nail, Marchetti-Vicenzi nail, Prevot pins)]. Unfallchirurg 1998; 101: $12-17$

[5] Scalea TM, Boswell SA, Scott JD et al. External fixation as bridge to intramedullary nailing for patients with multiple injuries and with femur fractures: damage control orthopedics. J Trauma 2000; 48: 613-621; discussion 621-623

[6] Salem $\mathrm{KH}$. Unreamed intramedullary nailing in distal tibial fractures. Int Orthop 2013; 37: 2009-2015

[7] Roussignol X, Sigonney G, Potage D et al. Secondary nailing after external fixation for tibial shaft fracture: risk factors for union and infection. A 55 case series. Orthop Traumatol Surg Res 2015; 101: 89-92

[8] Renner N, Regazzoni P, Babst R et al. [Initial experiences with the unreamed tibial nail]. Helv Chir Acta 1993; 59: 665-668

[9] Marshall PD, Saleh M, Douglas DL. Risk of deep infection with intramedullary nailing following the use of external fixators. J $R$ Coll Surg Edinb 1991; 36: 268-271

[10] Gartner ], Rudolph H. [Femoral shaft fracture. Treatment and results of 209 fractures]. Unfallchirurgie 1987; 13: 99-105

[11] Bhandari M, Adili A, Leone J et al. Early versus delayed operative management of closed tibial fractures. Clin Orthop Relat Res 1999; 368: 230-239
[12] Hofmann A, Dietz SO, Pairon P et al. The role of intramedullary nailing in treatment of open fractures. Eur J Trauma Emerg Surg 2015; 41: 39-47

[13] Wald ER. Risk factors for osteomyelitis. Am J Med 1985; 78 206-212

[14] Scolaro JA, Schenker ML, Yannascoli S et al. Cigarette smoking increases complications following fracture: a systematic review. J Bone Joint Surg Am 2014; 96: 674-681

[15] Dzupa V, Dzupová O, Bendová E et al. [Infectious complications of surgically treated musculoskeletal injuries: review of risk factors and etiological agents in years 2000-2005]. Klin Mikrobiol Infekc Lek 2007; 13: 242-247

[16] Santolini E, West R, Giannoudis PV. Risk factors for long bone fracture non-union: a stratification approach based on the level of the existing scientific evidence. Injury 2015; 46 (Suppl 8): S8-S19

[17] Malik $\mathrm{MH}$, Harwood $\mathrm{P}$, Diggle $\mathrm{P}$ et al. Factors affecting rates of infection and nonunion in intramedullary nailing. J Bone Joint Surg $\mathrm{Br}$ 2004; 86: 556-560

[18] Harris JC, Caesar DH, Davison C et al. How useful are laboratory investigations in the emergency department evaluation of possible osteomyelitis? Emerg Med Australas 2011; 23 : $317-330$

[19] Khan MH, Smith PN, Rao N et al. Serum C-reactive protein levels correlate with clinical response in patients treated with antibiotics for wound infections after spinal surgery. Spine J 2006; 6: 311-315

[20] Emara KM, Ghafar KA, Al Kersh MA. Methods to shorten the duration of an external fixator in the management of tibial infections. World J Orthop 2011; 2: 85-92

[21] Karargyris O, Romoudis P, Morassi LG et al. Distraction over nail using circular external fixation for septic pseudarthrosis of the tibia. J Long Term Eff Med Implants 2012; 22: 137-143

[22] Lin CC, Chen CM, Chiu FY et al. Staged protocol for the treatment of chronic tibial shaft osteomyelitis with Ilizarov's technique followed by the application of intramedullary locked nail. Orthopedics 2012; 35: e1769-e1774

[23] Morelli I, Drago L, George DA et al. Masquelet technique: myth or reality? A systematic review and meta-analysis. Injury 2016; 47 (Suppl 6): S68-S76

[24] Auregan JC, Begue T, Rigoulot G et al. Success rate and risk factors of failure of the induced membrane technique in children: a systematic review. Injury 2016; 47 (Suppl 6): S62-S67

Bibliografie

DOI https://doi.org/10.1055/s-0043-102295

OP-JOURNAL 2017; 33: 114-117 @ Georg Thieme Verlag KC Stuttgart · New York ISSN 0178-1715 